(n)

trobertivier Journal of Nonlinear Mathematical Physics

ISSN (Online): 1776-0852 ISSN (Print): 1402-9251

$P^{\text {rrumisis }}$ Journal Home Page: https://www.atlantis-press.com/journals/jnmp

\title{
On some connections between the Gompertz function and special numbers
}

Grzegorz Rządkowski, Wojciech Rządkowski, Paweł Wójcicki

To cite this article: Grzegorz Rządkowski, Wojciech Rządkowski, Paweł Wójcicki (2015) On some connections between the Gompertz function and special numbers, Journal of Nonlinear Mathematical Physics 22:3, 374-380, DOI:

https://doi.org/10.1080/14029251.2015.1079419

To link to this article: https://doi.org/10.1080/14029251.2015.1079419

Published online: 04 January 2021 
LETTER TO THE EDITOR

\title{
On some connections between the Gompertz function and special numbers
}

\author{
Grzegorz Rza̧dkowski* \\ Department of Finance and Risk Management, Warsaw University of Technology, Ludwika Narbutta 85 \\ 00-999 Warsaw, Poland \\ g.rzadkowski@wz.pw.edu.pl \\ Wojciech Rządkowski \\ Faculty of Physics, University of Warsaw, Hoża 69 \\ 00-681 Warsaw, Poland \\ Faculty of Electronics and Information Technology, Warsaw University of Technology, Nowowiejska 15/19 \\ 00-665 Warsaw, Poland \\ wojciech.rzadkowski@gmail.com \\ Paweł Wójcicki \\ Faculty of Mathematics and Computer Science, Warsaw University of Technology, Koszykowa 75 \\ 00-662 Warsaw, Poland \\ p.wojcicki@mini.pw.edu.pl
}

Received 14 March 2015

Accepted 3 June 2015

\begin{abstract}
In the present paper we show that the Gompertz function, the Fisher-Tippett and the Gumbel probability distributions are related to both Stirling numbers of the second kind and Bernoulli numbers. Especially we prove for the Gumbel probability density function an analog of the Grosset-Veselov formula which connects 1-soliton solution of the KdV equation with Bernoulli numbers.

Keywords: Gompertz function; Gumbel distribution; Bernoulli numbers; Stirling numbers of the second kind.
\end{abstract}

2000 Mathematics Subject Classification: 34A34, 11B68, 11B73

\section{Introduction}

The differential equation defining the Gompertz function is as follows

$$
u^{\prime}(t)=q u \log \frac{u_{\max }}{u}, \quad u(0)=u_{0}>0,
$$

where $t$ is time, $u=u(t)$ is an unknown function, $q, u_{\max }$ are constants. Constant $u_{\max }$ is called the saturation level. The integral curve $u(t)$ fulfilling condition $0<u(t)<u_{\max }$ is known as the Gompertz function.

*corresponding author 
Equation (1.1) is the first order ordinary differential equation which is easily solved by the method of the separation of variables. After solving it we get the following formula for the Gompertz function

$$
u(t)=u_{\max } e^{-c e^{-q t}},
$$

where constant $c$ appears in the integration process and is connected with the initial condition $u(0)=$ $u_{0}=u_{\max } e^{-c}$, therefore $c=\log \frac{u_{\max }}{u_{0}}$.

If $u_{\max }=1$ then (1.2) is the cumulative distribution function for the Fisher-Tippett distribution. A particular case of the Fisher-Tippett distribution (for $c=q=1$ ) is the Gumbel distribution with the probability density function (pdf): $g(t)=e^{-e^{-t}} e^{-t}$.

The Gumbel distribution has some application in physics. For example, Itoh, Mahmoud and Takahashi [8] consider a discrete model of wave propagation. They consider the wave propagation as motion of an ensemble of particles, each of them jumping forward with certain probability. The distribution of the quantity $\frac{L-k \ln k}{k}$, where $L$ is the wavelength and $k$ - number of particles is proven to approach Gumbel distribution for very large number of particles. This result corresponds to propagation of uniform soliton.

This idea is further explored by Itoh and Mahmoud [9] as they study the Moran population model. An ensemble of $k$ gametes is considered; each of gametes can die and produce a new gamete of age 0 . The probability of such evolution depends on gamete's age. Authors conclude that for large $k$ Gumbel distribution approximates $\frac{M-k \ln k}{k}$, where $M$ is the maximal age of the population.

Gumbel distribution is also of importance in geophysics. It was used to study extreme river floods [1,11,13], seismicity in Eurasia [3], occurence of extreme meteorogical events [10] or the level of Aegan and Ionian seas [15].

The Gompertz curve is also directly used for modelling different phenomena in biophysics, biology, medicine and others (see Stauffer et al [14], Waliszewski and Konarski [16]).

The paper is organized as follows. In Sec. 2 we recall some properties of Stirling numbers of the second kind and Bernoulli numbers which we use later. In Sec. 3 we show our main results concerning the Gompertz function and Gumbel's distribution. The paper is concluded in Sec. 4.

\section{Properties of special numbers}

By $\left\{\begin{array}{l}n \\ k\end{array}\right\}$ we denote the Stirling number of the second kind (number of ways of partitioning a set of $n$ elements into $k$ nonempty subsets; see Graham et al. [6]). It is set $\left\{\begin{array}{l}n \\ 0\end{array}\right\}=0$ for $n>0,\left\{\begin{array}{l}0 \\ 0\end{array}\right\}=1$, $\left\{\begin{array}{l}n \\ k\end{array}\right\}=0$ for $k>n$, or $k<0$. Let us recall that the numbers fulfill

$$
\begin{aligned}
& \left\{\begin{array}{l}
n \\
k
\end{array}\right\}=\frac{1}{k !} \sum_{j=0}^{k}(-1)^{k-j}\left(\begin{array}{c}
k \\
j
\end{array}\right) j^{n}=\frac{1}{k !} \sum_{j=0}^{k}(-1)^{j}\left(\begin{array}{l}
k \\
j
\end{array}\right)(k-j)^{n}, \\
& \left\{\begin{array}{c}
n+1 \\
k
\end{array}\right\}=k\left\{\begin{array}{l}
n \\
k
\end{array}\right\}+\left\{\begin{array}{c}
n \\
k-1
\end{array}\right\},
\end{aligned}
$$

and appear in the Taylor expansion

$$
\frac{\left(e^{w}-1\right)^{k}}{k !}=\sum_{n=k}^{\infty}\left\{\begin{array}{l}
n \\
k
\end{array}\right\} \frac{w^{n}}{n !} .
$$


By $B_{n}(n=0,1,2, \ldots)$ we denote the $n$th Bernoulli number. The Bernoulli numbers have the following exponential generating function (see Duren [5])

$$
B_{0}+B_{1} z+B_{2} \frac{z^{2}}{2 !}+\cdots=\frac{z}{e^{z}-1}, \quad|z|<2 \pi .
$$

It is well known that $B_{n}$ vanishes for odd $n \geq 3$. The numbers are rational and they appear in relations such that

$$
\sum_{k=1}^{m-1} k^{n}=\frac{1}{n+1} \sum_{j=0}^{n}\left(\begin{array}{c}
n+1 \\
j
\end{array}\right) B_{j} m^{n+1-j}, \quad m, n \geq 1,
$$

or

$$
\sum_{k=1}^{\infty} \frac{1}{k^{2 n}}=(-1)^{n+1} \frac{2^{2 n-1} \pi^{2 n}}{(2 n) !} B_{2 n}, \quad n=1,2, \ldots
$$

The first few nonzero Bernoulli numbers are as follows

$$
B_{0}=1, B_{1}=-\frac{1}{2}, B_{2}=\frac{1}{6}, B_{4}=-\frac{1}{30}, B_{6}=\frac{1}{42}, B_{8}=-\frac{1}{30}, B_{10}=\frac{5}{66}, B_{12}=-\frac{691}{2730} .
$$

Since

$$
\frac{1}{e^{z}+1}=\frac{e^{z}+1-2}{e^{2 z}-1}=\frac{1}{e^{z}-1}-\frac{2}{e^{2 z}-1}=\frac{1}{z} \cdot \frac{z}{e^{z}-1}-\frac{1}{z} \cdot \frac{2 z}{e^{2 z}-1}
$$

we infer from (2.3) that

$$
\begin{aligned}
\frac{1}{e^{z}+1} & =\frac{1}{z} \sum_{n=0}^{\infty} B_{n} \frac{z^{n}}{n !}-\frac{1}{z} \sum_{n=0}^{\infty} B_{n} \frac{2^{n} z^{n}}{n !}=\sum_{n=1}^{\infty}\left(B_{n} \frac{z^{n-1}}{n !}-B_{n} \frac{2^{n} z^{n-1}}{n !}\right) \\
& =\sum_{n=0}^{\infty} \frac{B_{n+1}\left(1-2^{n+1}\right)}{(n+1) !} z^{n}
\end{aligned}
$$

for $|z|<\pi$.

\section{Main results}

Theorem 3.1. If $u(t)$ is a solution of equation (1.1) then its $n$th derivative is given by the formula

$$
u^{(n)}(t)=q^{n} \sum_{k=1}^{n}(-1)^{n-k}\left\{\begin{array}{l}
n \\
k
\end{array}\right\} u \log ^{k} \frac{u_{\max }}{u}
$$

Proof. We will proceed by induction. For $n=1$ formula (3.1) transforms into (1.1), thus is true. Let us suppose that for a positive integer $n$ formula (3.1) holds. Then using the chain rule and identity 
(2.2) we get

$$
\begin{aligned}
& u^{(n+1)}(t)=q^{n} \frac{d}{d t} \sum_{k=1}^{n}(-1)^{n-k}\left\{\begin{array}{l}
n \\
k
\end{array}\right\} u \log ^{k} \frac{u_{\max }}{u} \\
& =q^{n} \sum_{k=1}^{n}(-1)^{n-k}\left\{\begin{array}{l}
n \\
k
\end{array}\right\}\left(\log ^{k} \frac{u_{\max }}{u}-k \log ^{k-1} \frac{u_{\max }}{u}\right) q u \log \frac{u_{\max }}{u} \\
& =q^{n+1} \sum_{k=1}^{n}(-1)^{n+1-k}\left\{\begin{array}{l}
n \\
k
\end{array}\right\}\left(k u \log ^{k} \frac{u_{\max }}{u}-u \log ^{k+1} \frac{u_{\max }}{u}\right) \\
& =q^{n+1}\left[(-1)^{n} u \log \frac{u_{\max }}{u}+\sum_{k=2}^{n}(-1)^{n+1-k}\left(k\left\{\begin{array}{l}
n \\
k
\end{array}\right\}+\left\{\begin{array}{c}
n \\
k-1
\end{array}\right\}\right) u \log ^{k} \frac{u_{\max }}{u}\right. \\
& \left.+u \log ^{n+1} \frac{u_{\max }}{u}\right]=q^{n+1} \sum_{k=1}^{n+1}(-1)^{n+1-k}\left\{\begin{array}{c}
n+1 \\
k
\end{array}\right\} u \log ^{k} \frac{u_{\max }}{u}
\end{aligned}
$$

Let us denote by $P_{n}(u)$ the right hand side of (3.1)

$$
P_{n}(u)=q^{n} \sum_{k=1}^{n}(-1)^{n-k}\left\{\begin{array}{l}
n \\
k
\end{array}\right\} u \log ^{k} \frac{u_{\max }}{u}, \quad n=1,2,3, \ldots
$$

and $P_{0}(u)=u$.

Remark 3.1. The Bell polynomials are defined as $B_{n}(x)=\sum_{k=1}^{n}\left\{\begin{array}{l}n \\ k\end{array}\right\} x^{k}$ (see Comtet [4]). Thus function $P_{n}(u)$ can be expressed in terms of the Bell polynomials as follows

$$
P_{n}(u)=q^{n} \sum_{k=1}^{n}(-1)^{n-k}\left\{\begin{array}{l}
n \\
k
\end{array}\right\} u \log ^{k} \frac{u_{\max }}{u}=(-q)^{n} u \sum_{k=1}^{n}\left\{\begin{array}{l}
n \\
k
\end{array}\right\}\left(-\log \frac{u_{\max }}{u}\right)^{k}=(-q)^{n} u B_{n}\left(\log \frac{u}{u_{\max }}\right)
$$

Now we introduce the exponential generating function (e.g.f.) for $\left\{P_{n}(u)\right\}$

$$
G(u, z)=P_{0}(u)+P_{1}(u) z+P_{2}(u) \frac{z^{2}}{2 !}+P_{3}(u) \frac{z^{3}}{3 !}+\cdots
$$

In order to find a closed form formula for e.g.f. (3.2) observe that $G(u(t), z)$ is the Taylor series expansion of the function $u(t+z)$ at point $t$. Therefore using formula (1.2) we get

$$
G(u(t), z)=u(t+z)=u_{\max } e^{-c e^{-q(t+z)}}=u_{\max }\left(e^{-c e^{-q t}}\right)^{e^{-q z}} .
$$

From (3.3) it follows that

$$
G(u, z)=u_{\max }\left(\frac{u}{u_{\max }}\right)^{e^{-q z}}
$$

Theorem 3.2. For the Gumbel's pdf $g(t)=e^{-e^{-t}} e^{-t}$ the following formula holds

$$
\int_{-\infty}^{\infty}\left(\frac{d^{k-1}}{d t^{k-1}}\left(e^{-e^{-t}} e^{-t}\right)\right)^{2} d t=(-1)^{k} \frac{B_{2 k}\left(1-2^{2 k}\right)}{2 k}
$$

where $k=1,2, \ldots$ 
Proof. Our aim is to compute integral $p_{n}=\int_{0}^{u_{\max }} P_{n}(u) d u, n=0,1,2, \ldots$ By (3.4) the e.g.f. for $\left\{p_{n}\right\}$ is

$$
\begin{aligned}
p_{0}+p_{1} z+p_{2} \frac{z^{2}}{2 !}+ & p_{3} \frac{z^{3}}{3 !}+\cdots=\int_{0}^{u_{\max }} G(u, z) d u=u_{\max } \int_{0}^{u_{\max }}\left(\frac{u}{u_{\max }}\right)^{e^{-q z}} d u \\
& =\left.u_{\max }^{2} \cdot \frac{1}{e^{-q z}+1}\left(\frac{u}{u_{\max }}\right)^{e^{-q z}+1}\right|_{0} ^{u_{\max }}=\frac{u_{\max }^{2}}{e^{-q z}+1} .
\end{aligned}
$$

Using formula (2.4) we obtain

$$
\frac{1}{e^{-q z}+1}=\sum_{n=0}^{\infty} \frac{B_{n+1}\left(1-2^{n+1}\right)}{(n+1) !}(-q z)^{n}=\sum_{n=0}^{\infty} \frac{(-q)^{n} B_{n+1}\left(1-2^{n+1}\right)}{n+1} \cdot \frac{z^{n}}{n !}
$$

and then comparing coefficients of $z^{n} / n$ ! in (3.6) and (3.7) we get

$$
p_{n}=\int_{0}^{u_{\max }} P_{n}(u) d u=\frac{(-q)^{n} B_{n+1}\left(1-2^{n+1}\right)}{n+1} \cdot u_{\max }^{2}
$$

Substituting in the integral(3.8) variable $t, u(t)=u_{\max } e^{-c e^{-q t}}$ we get

$$
\int_{0}^{u_{\max }} P_{n}(u) d u=\int_{-\infty}^{\infty} u^{(n)}(t) u^{\prime}(t) d t=\frac{(-q)^{n} B_{n+1}\left(1-2^{n+1}\right)}{n+1} \cdot u_{\max }^{2}
$$

If $n$ is an odd number $n=2 k-1$ then integrating in (3.9) $k-1$ times by parts we have

$$
(-1)^{k-1} \int_{-\infty}^{\infty}\left(u^{(k)}(t)\right)^{2} d t=\frac{(-q)^{2 k-1} B_{2 k}\left(1-2^{2 k}\right)}{2 k} \cdot u_{\max }^{2}
$$

or

$$
\int_{-\infty}^{\infty}\left(u^{(k)}(t)\right)^{2} d t=(-1)^{k} \frac{q^{2 k-1} B_{2 k}\left(1-2^{2 k}\right)}{2 k} \cdot u_{\max }^{2}
$$

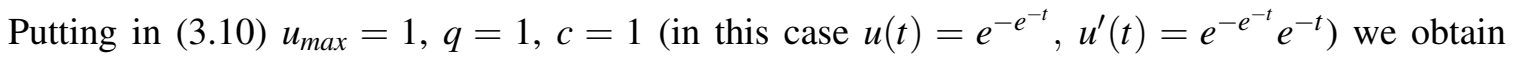
formula (3.5).

Remark 3.2. For the Gumbel's pdf the formula (3.5) can be seen as an analog of the GrossetVeselov formula

$$
B_{2 k}=\frac{(-1)^{k-1}}{2^{2 k+1}} \int_{-\infty}^{+\infty}\left(\frac{d^{k-1}}{d x^{k-1}} \frac{1}{\cosh ^{2} x}\right)^{2} d x,
$$

which has been demonstrated in [7]. For another proofs of (3.11) see [2] and [12]. Formula (3.11) shows the connection between 1-soliton solution of the KdV equation and the Bernoulli numbers.

We may also prove some explicit formulae, expressing Bernoulli numbers in terms of Stirling numbers of the second kind or binomial numbers. In order to do this let us first observe that integrating by parts we get the following recurrence formula 


$$
\int_{0}^{u_{\max }} u \log ^{n} \frac{u_{\max }}{u} d u=\int_{0}^{u_{\max }}\left(\frac{u^{2}}{2}\right)^{\prime} \log ^{n} \frac{u_{\max }}{u} d u=\frac{n}{2} \int_{0}^{u_{\max }} u \log ^{n-1} \frac{u_{\max }}{u} d u
$$

which by $\int_{0}^{u_{\max }} u d u=u_{\max }^{2} / 2$ leads to

$$
\int_{0}^{u_{\max }} u \log ^{n} \frac{u_{\max }}{u} d u=\frac{n !}{2^{n+1}} \cdot u_{\max }^{2} .
$$

In view of the definition of polynomial $P_{n}(u)$ and using (3.12) we can rewrite formula (3.8) as

$$
u_{\max }^{2} q^{n} \sum_{k=1}^{n}(-1)^{n-k}\left\{\begin{array}{l}
n \\
k
\end{array}\right\} \frac{k !}{2^{k+1}}=\frac{(-q)^{n} B_{n+1}\left(1-2^{n+1}\right)}{n+1} \cdot u_{\max }^{2},
$$

i.e.,

$$
\sum_{k=1}^{n}(-1)^{k}\left\{\begin{array}{l}
n \\
k
\end{array}\right\} \frac{k !}{2^{k+1}}=\frac{B_{n+1}\left(1-2^{n+1}\right)}{n+1} .
$$

Expressing in (3.13) the Stirling numbers by binomial numbers (2.1) we get

$$
\sum_{k=1}^{n} \frac{1}{2^{k+1}} \sum_{j=0}^{k}(-1)^{k-j}\left(\begin{array}{l}
k \\
j
\end{array}\right) j^{n}=\frac{B_{n+1}\left(1-2^{n+1}\right)}{n+1} .
$$

\section{Conclusions and further work}

We investigated some connections between the Gompertz function and special numbers (the Stirling numbers of the second kind and the Bernoulli numbers). We showed formula (3.1), involving Stirling numbers of the second kind, which expresses the $n$th derivative of the Gompertz function by this function itself. In a particular case $\left(u_{\max }=1\right)$ the Gompertz function is the Fisher-Tippett cumulative distribution function. A particular case of the Fisher-Tippett distribution (for $c=q=1$ ) is the Gumbel (standard) distribution.

Then we proved integral formula (3.5, Theorem 3.2), which shows a connection between the Gumbel pdf and Bernoulli numbers. In fact in the proof of Theorem 3.2 such connection is proved slightly more generally, for the Fisher-Tippett pdf and the Gompertz function. Formula (3.5) can be seen as an analog of the Grosset-Veselov integral formula which connects 1-soliton solution of the $\mathrm{KdV}$ equation with Bernoulli numbers.

We get also in Sec. 3 some formulae expressing Bernoulli numbers in terms of Stirling numbers of the second kind or binomial numbers.

The approach used here could be applied for other functions or even for systems of nonlinear differential equations like the Lotka-Volterra model.

\section{References}

[1] A. Asad, M. Ahmeduzzaman, S. Kar, A. Khan, N. Rahman and S. Islam, Flood frequency Modeling using Gumbels and Powells method for Dudhkumar river, Journal of Water Resources and Ocean Science 2(2) (2013) 25-28. 
[2] K.N. Boyadzhiev, A note on Bernoulli polynomials and solitons, J. Nonlinear Math. Phys. 14 (2007) 174-178.

[3] Paul W. Burton, Seismic risk in southern Europe through to India examined using Gumbel's third distribution of extreme values, Geophys. J. Int. 59 (2) (1979) 249-280.

[4] L. Comtet, Advanced Combinatorics (D. Reidel Publishing Co., Dordrecht, 1974).

[5] P. Duren, Invitation to Classical Analysis (American Mathematical Society, Providence RI, 2012).

[6] R. L. Graham, D. E. Knuth and O. Patashnik, Concrete Mathematics. A Foundation for Computer Science (Addison-Wesley Company, Inc., Reading MA, 1994).

[7] M-P. Grosset and A.P. Veselov, Bernoulli Numbers and Solitons, J. Nonlinear Math. Phys. 12 (2005) 469-474.

[8] Y. Itoh, H. M. Mahmoud, D. Takahashi, A Stochastic Model for Solitons, Random Struct. Algor. 24 (2004) 51-64

[9] Y. Itoh and H. M. Mahmoud, Age statistics in the Moran population model, Statist. Probab. Lett. 74 (2005) 21-30.

[10] H. A. Loaiciga and R. B. Leipnik, Analysis of extreme hydrologic events with Gumbel distributions: marginal and additive cases, Stoch. Env. Res. Risk A. 13 (1999) 251-259.

[11] M. K. Mukherjee, Flood Frequency Analysis of River Subernarekha, India, Using Gumbels Extreme Value Distribution, International Journal of Computational Engineering Research 03 (2013).

[12] G. Rza̧dkowski, Bernoulli numbers and solitons - revisited, J. Nonlinear Math. Phys. 17 (2010) 121126.

[13] O. Solomon and O. Prince, Flood Frequency Analysis of Osse River Using Gumbels Distribution, Civil and Environmental Research 10 (2013).

[14] D. Stauffer et al, Biology, Sociology, Geology by Computational Physisists (Monograph Series on Nonlinear Science and Complexity Vol. 1, Elsevier 2006).

[15] M. N. Tsimplis and D. Blackman, Extreme Sea-level Distribution and Return Periods in the Aegean and Ionian Seas, Estuarine, Coastal and Shelf Science 44 (1997) 79-89.

[16] P. Waliszewski and J. Konarski, A Mystery of the Gompertz Function, in: Gabriele A. Losa et al (eds.), Fractals in Biology and Medicine (Birkhäuser Verlag, 2005), 277-286. 